\title{
New Explorer for Communication of Postgraduate and Mentor in New Media Background
}

\author{
Lian-Xia JIANG ${ }^{1, a,{ }^{*}}$ \\ ${ }^{1}$ Graduate School, South China University of Technology, Guangzhou, Guangdong Province, China \\ axjiang@scut.edu.cn \\ ${ }^{*}$ Corresponding author
}

Keywords: New media, Postgraduate, Communication.

\begin{abstract}
The enormous gathering of cyberspace promotes the rapid development of the new media concept and technology, which has obvious effect in the infiltration of daily behaviors of teachers and students, the communication between mentors and postgraduate gets more closely in such condition, but easily to be neglected. By studying the current situation and the positive signification of the communication of postgraduate and mentor in new media background, some suggestions and measures are presented to improve the communication quality of teachers and students.
\end{abstract}

\section{Introduction}

Wechat, Weibo, Quora, and Live net platform are gradually to be one part of people's work and life, new media network technology innovation has been changed into the concept of innovation, interaction, and sharing from reading writing, people has a new experience in the thinking mode, working ways and life and entertainment etc. The advantages that convenience, fast and big data has made the new media connect with the political, economic, culture, technology, and education etc. there is no exception for the development of colleges and universities, the network interconnection mode has been deeply embraced in teaching and management, and at meanwhile has profound influence on the daily living and interpersonal communication of teachers and students. As a part of colleges and universities, postgraduate have the characteristics of stronger academic ability and higher comprehensive quality, the communication between mentors and postgraduate, especially the progress of academic exchanges and daily communication based on new media platform will directly affect the growth trajectory of the high-quality talents, and then it is related to the social development and the evolution of data-intensive and network. The paper discussed the relationship status, significance and suggestions of postgraduate and mentors based on actual research in new media background.

\section{The Research on Current Relationship between Postgraduates and Supervisors under New Medias}

\section{The Contradiction between The Equality of Students And Teachers That The Internet Medias Promote and Seniority In Them}

There are diversified and vibrant voices on the internet and many aspects shown, which mainly includes few features: The first one is that people at different ages all use the new medias without age limit to access the internet. The second one is that people use the new medias at any time and any places. There is no line among information and both the professional and the non-professional all make information. The new medias with these features promote the free and equal relationship that is close to a relaxing and flexible relationship. The philosopher in West Han Dynasty remarked: "The teacher is the model for people.” As ancient Chinese proverb goes, "once a teacher, always a father”. We can tell "respecting teachers and valuing knowledge" is an important part in Chinese traditional culture. Therefore, the relationship between students and teachers has a sort of rigid and rigorous 
definition. In other words, most of postgraduate supervisors are excellent academic elites and pioneers in their field and postgraduates adore their supervisors naturally for their academic achievements. The gap for ability and knowledge and adoring thing produce a certain distance between postgraduates and supervisors. The free and relaxing environment created by the internet media can't bring them together for a short time. Thus, in most cases, There is an obvious contradiction between the equality of students and teachers that the internet medias promote and seniority in them.

During doing the research, 58\% of postgraduates think that the internet is the common platform for them to interact with others but only $8 \%$ of them will choose to use the internet communication application to communicate with their supervisors. At the same time, only $28 \%$ of postgraduates think the new internet platform can narrow the distance between them and their supervisors and only $24 \%$ of postgraduates are willing to communicate with their supervisors on the internet media platforms. We can tell there are difficulties of communicating on the internet for postgraduates and supervisors to solve. The difficulties include: The first one is that postgraduates don't dare or like to communicate with their supervisors on the new medias, which maybe is caused by the seniority in them to some degree. What's more, the internet social circle created by postgraduates is the source for them to get pleasure and happiness after class and also an important space for them to get rid of complicated experiments and researches and fill the emptiness of life. Thus, postgraduates have already divided the study and life into two separated parts. And they care more about the pleasure they can get from the internet medias. Postgraduates are not willing to let their supervisors in and break this balance between these two parts. The communication between postgraduates and their supervisors are often concerning their study. When postgraduates and their supervisors can't eradicate the seniority in them, they just use the internet medias as tools. The interaction like this may help them convey tasks but also ignores the real meaning of interaction of postgraduates and their supervisors. And they can't really know each other and get any progress in improving their relationship. This progress not only is about the development of study but also affects postgraduates and their supervisors' characters.

\section{The Contrast Formed between The Internet Thinking of Life And The Traditional Concept, Teachers' Attitudes Are Far from That of Students to the New Media}

According to the survey, 89\% of postgraduates are using the Internet social networking platform every day. The Internet new media has become an indispensable part of postgraduate's daily life. postgraduate tutors concentrate in the age between 40 to 60 years old, the busy daily work and stable life philosophy make them be shallow in the new field of communication and understanding of technology, and pay little attention to a variety of network platform, resulting in the different attitude of both teachers and students of the new media, which is mainly reflected in the several aspects:

First of all, it is the basic idea of new media. With the development of the Internet, new media technology and innovation in the Internet era have undergone changes. Young people's thinking has created great momentum. As a member of this group, postgraduate is not only the creator of the new media era, but also an important promoter of its application. Most postgraduates have been well adapted to the multiline and multi-dimensional state of interaction, sharing and blending of new media, and they are very willing to connect media applications with daily learning, life travel, dating, love and internship. While the tutors' attitude to the media, most of them are concerned with data integration, acquisition and communication, and lack of interaction and sharing. At the same time, the traditional and cautious attitude makes tutors not willing to show their willingness to work and life in the media, and the resonance between postgraduates and mentors in media concept is less.

The second is the time for the use of new media. Brushing micro-blog, brushing WeChat, and the Q group of forums are also a "part of the matter" for young postgraduates, while most traditional tutors take the new media communication as a kind of "external things", therefore, the length of time span of interpersonal communication appeared on two different states in the virtual space. On young postgraduates, the time occupied by new media can cover public life and private life, day and night, and the time line is not obvious. The probability of media involvement in life is relatively high. On the 
contrary, because of the relatively low degree of acceptance of the postgraduate tutors, it is obvious that the time span of media use is shorter and the frequency of interaction is lower.

Then, it is on the platform of the application of new media. On one hand, the postgraduates are more skilled than tutors in the switch and integration of PC terminal (computer) and mobile terminal (mobile phone), the skills of input information, discriminant information and output information skills are clearer and more efficient. On the other hand, more platforms are contacted and regarded as the frequent use of media by postgraduates, according to the survey, application of the tutorial on media basically concentrated in the two part of QQ and WeChat, but in addition to QQ and WeChat, the postgraduates are also more active in the forum, video and other forms of media, including Know almost, Baidu, Wikipedia, Youku video, Iqiyi video, and also including many mobile terminal APP involving many friends, shopping, sports, cultural and creative content..

The Content of Network Communication Tends to be Utilitarian and Practical, which Compresses The Space and Time of Humanistic Interaction between Teachers and Students.

By comparisons of questionnaire survey, it shows that top3 of the daily network communication of postgraduate are 'hobbies and interests' (75.05\%), 'life anecdotes' (54.36\%), 'academic and professional' (41.02\%), and the top3 of network communication between postgraduates and mentors are 'academic and professional' (91.06\%), 'work practice' (50.04\%) and 'life anecdotes' (20.26\%). It is obviously shows that the communication content of teachers and students is more purposeful and unicity, the media communication platform not only plays the role of distance extend between teachers and students, but also the more embodies in tools. In fact, postgraduates tend to notice the development of perceptual factor in network media, and prefer to pay more attention to the feeling interaction in life and the recognition and improvement of personal cultural perception, such as the activity that to continually update the life state and Chicken Soup on Weblog and Wechat, by releasing the individual perception to external object and inward word, or draw other people's ideas to complete the communication of network. But the current state is that mentors and students are often ignore the convenience and rich content provided by the use of internet media which can be used to enhance communication, also the circumstances often occurs that one shield other one, which is cutting off the communication bridge.

The emergence of new media, completing the task that big data and high-tech means required for economic production and development, besides, the greatest contribution is providing the enormous humanities content. The postgraduate training, need the professional competence, and also need so much more in the strong foundation of humanistic literacy. Paying attention to the humanistic and improve the knowledge appreciation and self-cultivation should be one important part in postgraduate growth. By culture communication of network, on one hand, we can feel the cultural difference, offset weakness, on the other hand, it can solve troubles and find solution to problems, and also it can make people more social and have more sense of mission through human spirit edification. As the saying goes, mentor of postgraduates are not only the 'Scribe', but also the 'Teacher', which has self-evident effect in the promotion of humanistic quality of postgraduates. By the training of the mentor-apprentice style, the best training result not only achieve 'similarity in form' (research direction and research method), but also achieve 'similarity in spirit' (humanistic spirit and dedication of science research). Therefore, the postgraduate self enhancement or mentor's teaching method, the more involves in internet is people-to people ties, it is necessary to cultivate humanistic feelings. The compressed space urgently needs to be released, or it's inevitable that there will be postgraduate malignant event due to psychological problems and communication problems between teachers and students.

\section{The Real Meaning of Interaction Between Supervisors And Postgraduates Under New Medias}

So-called interaction between people is an activity for them to talk to and understand others via a series of medias so as to change the world and change themselves. New medias is the new-born things 
of the times and serve as the important bridges for supervisors and postgraduates. The interactions on the new medias between supervisors and postgraduates will improve their communications on profession, emotion and humanity.

\section{Focusing on Interaction on New Medias is the Important Step for Supervisors to Nurture Students and Postgraduates to Achieve Their Academic Goal}

According to researches by scholars, postgraduates' research achievements are the results produced by their individual recognition, behavior and environments they are in, among which there are three important preconditions consisting of how postgraduate are loyal to their study, how passionate supervisors are to give instructions and how often supervisors interact with postgraduates. All these affect postgraduates' research achievements. We can tell we can't make and implement the postgraduates' programs, training systems and teaching plans neither only considering considering how passionate postgraduates are to their major nor only considering supervisors' academic authority, professional charm and training skills. Only adopting the teaching model of interaction will supervisors and postgraduates match with each other's goal.

As mentioned before, postgraduates' study and spare time are gradually full of gradually new medias like WeChat, Weibo, Zhihu, Baidu Tieba, online video applications. Thus, as for supervisors, they can pay attention to postgraduates' action on the new medias and interact with them. They can first know postgraduates' interests on the study and how passionate they are to their study. Referring to this, they can find postgraduates' potentials and follow this effective instructive mechanism. In addition, they can find their postgraduates' misunderstandings and incorrect view on study and help them to correct in time. Then, they will know postgraduates' study conditions and encourage students to focus the study using common language indirectly. As for postgraduates, knowing how to use new medias to interact with supervisors first can let them discuss problems at anytime and any places. The discussions between them don't only happen in classrooms, offices or labs anymore. The interactions on new medias can not only extend the academic topics between them but also enrich knowledge and researches. In addition, new medias will make postgraduates often communicate with supervisors obviously and they have more opportunities to interact with supervisors so as to gain appreciation and attention, which help them to make progresses in study and have more chances to get good jobs.

\section{Focusing on New Media Communication is the Best Place For Both The Tutors and The Graduate Students to Break Down The Discourse and Confide}

In daily communication practice, although the postgraduates have entered the stage of elite education, their knowledge level and quality has reached a better development, influenced by traditional cultural ideas, the sense of hierarchy of teachers and students is still firm. Most of the teachers who are increasingly focused on academic and administrative work enjoy the satisfaction of this mentoring relationship, because the symbol capital of this "graduate tutor" is an important manifestation of their respect and recognition and self value realization. [Hu Tianyou. Partner: tutor and a possible [J]. degree and graduate education graduate, 2013 (4): 8.] therefore, this gap results that in the interaction process of imparting knowledge, most tutor are dominant and graduate students are responsible for their responses. In the process of caring for life, postgraduate scruples that their self identity will make the topic "not up to the table", which leads to more superficial greetings.

Some scholars have pointed out that in the interpersonal interaction of daily life, the physical embeddedness is the basic way to maintain a coherent sense of self identity. This kind of "communicative presence" which wants to get the necessary sense of achievement and energy, most are parallel or generation identity prevailed in lectures and discussions. Otherwise, the inappropriate speech and behavior in communication will expose the real idea or weakness of the heart, which will reverse the shaping of self identity. When people are in a state of "insecurity", it is not conducive to the depth and continuity of communication. The interpersonal interaction in the network space forms the "Absence" of the communication status, and avoids the direct contact of the body, which makes the identity, status, wealth and so many factors premise of achievement identity play a limited role, 
this is one of the reasons for the equality and liberty of new media communication. In the new media, when putting down the identity of the burden of "lower", the postgraduates have the courage to give tutor "message", dare to elaborate coverage of professional learning, emotional life, work practice, social opinion and other content ideas, and with interactive tutor, and essence of mining problem, which has also contributed to the intention of the graduate students to express their heart and express their feelings. When the tutors involved in new media and are willing to accept the concept of point of view, they will be able to absorb more from the network of the new culture, and understand the mental health of Postgraduates in the new era and daily behavior, so that we can further grasp the personality and emotional dynamics of students, and give them all dimensional care.

\section{Focusing on The Communication Way of New Media Is An Excellent Approach for Instructors and Postgraduates To Transfer Information, Conduct Communication and Enhance Their Humanistic Qualities with Rich Contents}

The important target of elite education is to improve the humanistic quality of an individual and to cultivate all-round postgraduates. For postgraduates, strengthening the cultivation of humanistic quality is to further pursue the treasure of human cultural spirit except the professional knowledge and skills, to form the profound understanding of the development of human science and technology civilization and to enhance the perceptual knowledge with the people-oriented and life-based attitudes towards happiness. From these perspectives, the new medium which acts as the object perceived, not only serves as a raw material conveyor but also shoulders the work of polish and rendering. Especially, it plays a crucial role in the communication between instructors and postgraduates and helps the exchange of humanistic feelings and mutual learning way to exert great efficiency.

First of all, the rich new media contents provide mentors and students with most choices in the theme of communication. Jaspers proposed that communication was the existing way of humans, a universal condition for people and an important form by which all people stepped on the common road. The common path must be started first by a common topic, and the same natures as industry, field and age make it easier for the communication to take place. The system of two-way choice gives both mentors and postgraduates a mutual understanding chance before their cooperation. Therefore, mentors and students have more or less similarities in political, historical, cultural and artistic aspects, in addition to professional knowledge. They are conducive to foster the perceptual and rational understanding of the human world, form a good relationship between instructors and students in some aspects and more importantly, improve humanistic qualities in sharing comments, through the discussion, forwarding and evaluation of the third website platform. Secondly, from the perspective of the fusion of horizon, the different cultural subjects have different cultural expectations distinctly, and hope to get different cultural field of fusion vision. Therefore, the relationship between students and mentors is formed by the collision and integration of their different cultural visions to some extent, which is the process of their vision superposition and reunification. And the process is always accompanied by the consideration of the spiritual world, and the concern of human nature, which is the very refining process of humanistic qualities. What's more, the extensity and randomness of new media provide students and mentors with more time and space in examining issues and expressing opinions. Postgraduates sometimes fail to talk about the topics thoroughly because of such factors as timidity, slow reaction at the scene or improper occasions during the face-to-face exchange with instructors. The rather superficial and unilateral conversation in the face-to-face communication can be avoided when people want to express their cultural views through the transferring forms like the links of texts, photos and videos or leave a message to share their views of social and cultural hotspots by the communication platform. At the same time, the quality of communication can be improved in the basis of more consideration and in-depth reply. Only in such process of humanistic exchanges, can we be able to get the spiritual comprehension and then cultivate personal moralities and good behaviors. 


\section{Some Suggestions on The Interaction between Tutors and Post-Graduates Under The Background of New Media}

\section{On the Tutor Level, We Should Accept the New Media in All Directions and Strengthen the Communication with Post-Graduates with the Help of the Platform.}

First of all, we should change the thinking according to the characteristics of the new media. On one hand, the tutor should accept the Internet as an important part of daily life, and take part in the change from offline concern and reality to online. On the other hand, we should understand the sharing characteristics of network, form the thinking of sharing power, be good at expressing their own opinions on different new media platforms, and become the makers or leaders of the community at the right time. We must understand the interactive characteristics of the network, form the thinking of interactive debates, and achieve different communication effects through interchanging views with graduate students, even colleagues and friends on some academic issues and network opinions.

Secondly, we should master the characteristics of the internet and change the role. In the communication of new media, tutors are no longer just the instructors of knowledge and subjects, but should also put themselves in the position of learners, not only lean from experts and authorities, but also from young netizens and postgraduates. Therefore, when we further clarify the characteristics of the Internet equality, the tutors should also lay down their burden and regard themselves as an ordinary netizen's identity, so that the probability of the listener will be higher than that of the instructor.

Then, we should understand the platform of the new media and strengthen the skills. With the development of new media, the sound and image characters have been very mature and universal, even develop towards the trend of artificial intelligence and scene. Video APP website, communication software, forum sharing and other basic new media forms should become the tutor's daily attention. In the process of professional communication or life communication, tutors should also grasp basic writing, image and dynamic sharing production skills, in order to attract the attention of postgraduates and peers, and further improve the possibility of communication.

Finally, we should look at the new media objectively and maintain the authority. Although the new media Internet advocates highly freedom and equality and canonizes sharing and interaction, as top-level instructors and designers of elite education, postgraduate tutors need to maintain necessary authority. On one hand, continue to strengthen the professional authority, continue to expand and further enhance the status of the industry in the professional field, continue to worship teacher occupation career sense of postgraduates, on the other hand, continue to create personality authority, whether online or offline, they all have to form the charm of German and academic personality through words or behavior, and form a subtle influence in the process of communication with graduate students.

\section{Postgraduates Should Improve Quality to Use Media and Interact with Supervisors on Proper Platforms}

First of all, postgraduates should improve their quality to use media. With the internet developing and changing, people should keep reasonable and acquire critical thinking and better ability to gain information in order to get acclimatized to changes of conditions and ideas and build a better social internet environment to gain best resources, which is so-called quality of media. Facing the complicated information on the internet, postgraduates need to maintain a critical attitude when choosing, understanding, questioning, assessing, creating and spreading the interaction subjects, information and tunnels, in which can they guarantee information is used effective by them and they are not kidnapped by the views on the internet and lost in the mass. Meanwhile, choosing the quality content to spread makes people have stronger desire to share and have more feedbacks so as to achieve the goal of interaction.

Secondly, postgraduates should learn to make use of medias properly. For online or offline or professional or life, no matter what, postgraduates' and supervisors interact with each other at a 
certain time or space. For supervisors, title assessment, administration works, seminars, daily courses and other works take most of their time and space. Thus, supervisors will choose the interaction subjects with obvious goals when facing real life or virtual world. Although supervisors need to update their knowledge for new media so as to keep up with the times, it is difficult for them to make full use of the new medias. Thus, postgraduates should choose popular medias that are easy to use, like WeChat, Weibo or forum. They can share their ideas and views with their supervisors in time through messages, group chat and forum. Besides, they should combine the dynamic and static technologies of internet, follow the humorous style and use the repost and share function especially so as to enrich the interaction contents.

\section{In Institutional Level, the Attitude Should be Tolerant to New Media Blend in the University Elite Education}

First of all, from the angle of overall institutions, the dynamics changes caused by the springing up of new media has been accepted by contemporary campus environment, colleges and universities have done a lot of guidance work on science tendentiousness in students participating in Internet interaction, and also laid a good foundation for creating a healthy cyberspace. On one hand, cyberspace, new media intellectual as the update of education tool, institutional decided to give great support om hardware, the mentors should firmly master techniques and reasonably apply them into teaching and academic activities; on the other hand new media plat form as the stretch of real life, the institution should pay attention to the occupation on the space, to make it be the new type possy of thinking education and quality education. However, seizing and occupation not means the blocking and joseki, especially for postgraduates and mentors who have active mind, they should be given more right of speaking and expressing, more viewpoint and opinions should be accepted, to build more bridge for communication will promote the revolution and development on institution, mentor and postgraduate.

Secondly, from a grassroots perspective, new media should be used as widely as they can to create communication groups of high-quality products. For example, in various colleges, subject group, mentor group, laboratory, through the mentor guidance, postgraduate management operation form, to form different topics of network communication groups, expand professional communication channels and cultural interest exchanges. This kind of "huddle" way with organization and authority, although face with the problem that existence time is short, persistence is low, but it only needs one brainstorming, the divergent opinions close dialogue, which can make a in-depth topic, then promotes the communication emotion expression, even get the solutions. From the point of view of the Poseidon effect (In a virtual game, if a person has a very attractive incarnation, and thus get the respect of others in the online world, then his behavior in real life will be more confident) For postgraduate who are not good at speaking, this form of communication will give them real opportunities and courage to express their inner feelings, and can also motivate them to perform well in daily practice. Therefore, paying attention to the construction of new media small group network will also improve the quality of communication between postgraduates and mentors.

\section{Acknowledgement}

This research was financially supported by the Guangdong Philosophy and Social Science "twelve Five Year" planning discipline co-construction project (No. GD14XJY06), and Guangdong Provincial Education Science Planning Project (Ethnic Education Project) (No. 2016JKDY01).

\section{References}

[1] The Ministry of Education Several Opinions on Further Strengthening and Improving Ideological and Political Education Graduate, 2010. 
[2] Q. P. HUANG, Research Status of Relationship between mentors and students, Youth and Society, 2016, No.12, p. 56.

[3] Y. A. YUAN, German Teacher Training Guide, People's Education Press, 2001.

[4] Y. M. TIAN, On the Role of Teachers in Postgraduates' Ideological and Political Education, China Electric Power Education, 2008, No.11, p. 36.

[1] X. M. WANG, Research on the Influence of Teacher-Students Communication in Postgraduate Education on Postgraduate Knowledge Output [D]. Huazhong University of Science and Technology, 2013.

[2] T. Y. HU, Partnership: A Possibility of the Relationship between Tutors and Graduate Students [J]. Academic Degrees \& Graduate Education, 2013 (4).

[3] X. WU, Cultural Dialogue and Visual Integration: Modernity View of the Relationship between Teachers and Graduate Students --- An Analytical Framework for Educational Anthropology [J]. Education and Teaching Research, 2011, 25 (7).

[4] A. NICHOLAS, H. F. JAMES, Connected: The Surprising Power of Our Social Networks and How They Shape Our Lives [M]. Beijing: China People's Publishing House.2013.

[5] E. Q. HONG, T. Y. HU, The Traditional Transcendence of the Relationship between Tutors and Graduate Students [J]. Contemporary Education Science, 2011 (3).

[6] Q. SUN, Q. F. HOU, The Construction of the Harmonious Relationship between Tutors and Graduate Students from the Perspective of Communication Theory [J]. Education Review, 2015 (2).

[7] W. W. LU, Study on the Relationship between College Tutors and Graduate Students from the Perspective of Communication [D]. South-Central University For Nationalities, 2010.

[8] H. JURGEN, The Theory of Communication Behavior: Behavior Rationality and Social Rationality [M]. Shanghai: Shanghai People's Publishing House, 2004.

[9] J. KARL, What is education? [M]. Beijing: Joint Publishing, 1991.

[10] Q. ZHOU, T. FENG, W. X. LIANG, The Exploration of the Relationship between Teachers and Graduate Students in the Network Age [J]. Academic Degrees \& Graduate Education, 2011 (4).

[11] J. K. CHEN, Thinking on the Construction of the Relationship between Teachers and Graduate Students from the Perspective of Cultural Feeding [J]. Academic Degrees \& Graduate Education, 2010 (9). 